TRANSACTIONS OF THE

AMERICAN MATHEMATICAL SOCIETY

Volume 279, Number 1, September 1983

\title{
GRAPHS WITH RELATIONS, COVERINGS AND GROUP-GRADED ALGEBRAS
}

\author{
BY \\ EDWARD L. GREEN ${ }^{1}$
}

\begin{abstract}
The paper studies the interrelationship between coverings of finite directed graphs and gradings of the path algebras associated to the directed graphs. To include gradings of all basic finite-dimensional algebras over an algebraically closed field, a theory of coverings of graphs with relations is introduced.
\end{abstract}

The object of this paper is to relate group gradings on algebras to coverings of a graph which is associated to the algebra. The linking of the theories allows one to relate purely algebraic questions to questions in algebraic topology, group theory or combinatorics.

In the representation theory of Artin algebras the association to each algebra of a finite directed graph, called the quiver of the algebra, has been a useful tool. The reason that the quiver of such an algebra is of interest is that there is a natural definition of representations of the quiver so that the category of representations of the quiver satisfying certain relations is equivalent to the category of finitely generated modules over the algebra. $§ 1$ gives a slight extension of these concepts to finitely generated algebras.

The main emphasis of the paper is to show that the theory of coverings of graphs with relations, introduced by C. Riedtmann [9] and expanded by P. Gabriel [2], and the theory of group-graded algebras are essentially the same. Although the original connection between coverings and gradings was inspired by the similarity of results for Z-graded Artin algebras [3,4] and P. Gabriel's announced results [2], the context of this paper is more general and deals with all finitely generated algebras over a field. We associate to each such algebra a finite directed graph which we still call a quiver of the algebra. We show that for each regular covering $\Gamma$ of a quiver $\Gamma_{0}$ of an algebra $\Lambda$, with certain prescribed restrictions, we get a $G$-grading of the algebra $\Lambda$, where $G$ is the automorphism group of the covering $\Gamma$ over $\Gamma_{0}$. Conversely, given a certain type of $G$-grading of $\Lambda$, where $G$ is a group, we construct a regular covering $\Gamma$ of the quiver $\Gamma_{0}$ of the algebra such that $G$ is isomorphic to the automorphism group of $\Gamma$ over $\Gamma_{0}$. Furthermore, if $\Gamma$ is a regular covering of $\Gamma_{0}$ with automorphism group $G$, we show that the category of representations of $\Gamma$ satisfying a certain set of relations is equivalent to the category of finite-dimensional graded $G$-modules. We

Received by the editors September 20, 1982.

1980 Mathematics Subject Classification. Primary 16A03, 57M10; Secondary 16A90, 05C20.

${ }^{1}$ The author gratefully acknowledges the partial support of the National Science Foundation. 
also show that universal coverings exist in an appropriate category of coverings of a fixed graph with relations.

In [7] we show how the results on Z-graded Artin algebras developed in [3, 4] can be changed to hold for general group-graded Artin algebras. In that paper we show how these results, together with the results of this paper, may be used to handle the general classification of which finite-dimensional algebras satisfying "zero relations" are of finite representation type.

To summarize this paper section by section: in $\S 1$, we detail the known correspondence between a finitely generated algebra and its quiver. We include these results for two reasons: namely, for notation and, more importantly, for the development of a point of view. In $\$ 2$ we introduce the category of coverings of a graph with relations and show that there are "universal coverings" in this category and also relate these results to categories of representations of the graphs with relations. $\S 3$ contains the main results mentioned above relating group gradings of a finitely generated algebra to coverings of the quiver of the algebra. Finally, $\$ 4$ contains an example and concluding remarks.

I am indebted to a number of mathematicians for helpful discussions that led me to these results. First I must thank Maurice Auslander and Idun Reiten for their interest and insight. I would also like to thank Dan Farkas, Charles Feustal and Robert Snider for their help. Finally, I would like to express my appreciation to the referee.

1. Finitely generated algebras and representations of graphs. Throughout this paper $k$ will denote a fixed field. We say a $k$-algebra $\Lambda$ is finitely generated if $\Lambda$ is a homomorphic image of a free associative $k$-algebra in a finite number of noncommuting variables. Note that a free associative $k$-algebra with $m$ noncommuting variables can be viewed as the tensor algebra $T_{k}(V)=\amalg_{i=0}^{\infty}\left(\otimes_{k}^{i} V\right)$ where $V$ is a $k$-vector space of dimension $m$. More generally, let $R$ be the product of $n$ copies of $k$; that is, $R=\prod_{i=1}^{n} k_{i}$ where $k_{i}=k$ for $i=1, \ldots, n$. We view $R$ as a $k$-algebra via the diagonal action. Let $M$ be a finitely generated $R$ - $R$-bimodule. Let $T_{R}(M)$ denote the tensor algebra $\amalg_{i=1}^{\infty}\left(\otimes_{R}^{i} M\right)$. Obviously, the free associative algebra is a special case of this construction by setting $n=1$.

It is immediate that $T_{R}(M)$ is a finitely generated $k$-algebra. Thus, every finitely generated $k$-algebra is a homomorphic image of some $T_{R}(M)$. Let $I$ be an ideal in $T_{R}(M)$ and $\Lambda=T_{R}(M) / I$. Let $\rho$ be a set of generators for $I$. Then the choice of $R, M$ and $\rho$ can be viewed in the same fashion as being given generators and relations of a finitely generated group. In particular $T_{R}(M)$ is a "free" object in the category $k$-algebras (see [5, Appendix]). As with generators of groups, there is no unicity in the choice of a ring surjection $T_{R}(M) \rightarrow \Lambda$ and, as with relations among the generators of a group, there is no unicity in the choice of generators $\rho$ of the ideal $I$.

We look more closely at the structures given above. Let $R=\prod_{i=1}^{n} k_{i}$ with $k_{i}=k$ for $i=1, \ldots, n$. Then, by the semisimplicity of $R \otimes_{k} R$, there are nonnegative integers $n_{i j}$ such that $M \cong \amalg_{i, j=1}^{n}\left({ }_{i} k_{j}\right)^{\left(n_{j i}\right)}$ as $R$-R-bimodules, where ${ }_{i} k_{j}=k_{i} \otimes_{k} k_{j}$ 
and $\left({ }_{i} k_{j}\right)^{\left(n_{i j}\right)}$ denotes the direct sum of $n_{j i}$-copies of ${ }_{i} k_{j}$. The (left) quiver of $T_{R}(M)$ is the finite directed graph $\Gamma$ such that the vertex set consists of $n$ vertices $v_{1}, \ldots, v_{n}$ and the arrow set consists of, for each pair of vertices $v_{i}, v_{j}$, exactly $n_{i j}$ arrows from $v_{i}$ to $v_{j}$. In general, if $\Gamma$ is a directed graph (not necessarily finite) we denote the vertex set of $\Gamma$ by $V(\Gamma)$ and the arrow set by $A(\Gamma)$. If $\Lambda=T_{R}(M) / I$, the (left) quiver of $\Lambda$ with respect to the presentation $T_{R}(M) \rightarrow \Lambda$ is the left quiver of $T_{R}(M)$.

Note that given a finite directed graph $\Gamma$ we may define the free path algebra, $T(\Gamma)$, associated to $\Gamma$ as follows. As a vector space, $T(\Gamma)$ has as a $k$-basis the set of directed paths in $\Gamma$ (of finite length). Here paths of length 0 are the vertices. Multiplication of directed paths is composition if defined, or 0 if they cannot be composed. (See [1].) If $\Gamma$ is the quiver of $T_{R}(M)$ then it is well known that the $k$-algebras $T_{R}(M)$ and $T(\Gamma)$ are isomorphic. If $\rho$ is a set of generators of an ideal $I$ in $T_{R}(M)$, viewing $T_{R}(M)$ as $T(\Gamma)$, the elements of $\rho$ are just $k$-linear combinations of directed paths in $\Gamma$.

Let $\Lambda=T(\Gamma) / I$ and let $\bmod (\Lambda)$ denote the category of finite-dimensional (over k) $\Lambda$-modules. Next we show how to describe $\bmod (\Lambda)$ as "representations of $\Gamma$ ". For later use we give a more general definition.

Let $\Gamma$ be a directed graph (not necessarily finite). A $k$-representation of $\Gamma$ is a tuple $X=\left(X_{v}, \alpha(a)\right)_{v \in V(\Gamma), a \in A(\Gamma)}$, where each $X_{v}$ is a finite-dimensional $k$ vector space, all but a finite number zero, and if $a$ is an arrow from vertex $v$ to vertex $w$ then $\alpha(a)$ is a $k$-linear map from $X_{v}$ to $X_{w}$. The category of $k$-representations of $\Gamma$, denoted $\operatorname{rep}(\Gamma)$, has as objects the $k$-representations of $\Gamma$ and as morphisms $B:\left(X_{v}, \alpha(a)\right) \rightarrow$ $\left(Y_{v}, \beta(a)\right)$ tuples $B=\left(B_{v}\right)_{v \in V(\Gamma)}$ such that each $B_{v}$ is a $k$-linear map from $X_{v}$ to $Y_{v}$ such that if $a$ is an arrow from vertex $v$ to vertex $w$ then $B_{w} \circ \alpha(a)=\beta(a) \circ B_{v}$.

Before defining what it means for a $k$-representation to satisfy a set of relations we need more terminology. If $t=\sum_{i=1}^{m} \mu_{i} p_{i}$ is a $k$-linear combination of directed paths in $\Gamma$ and $v, w$ are vertices in $\Gamma$, we let $c_{v, w}(t)$ be the $(v, w)$-component of $t$ where $c_{v, w}(t)=\sum_{j=1}^{m^{\prime}} \mu_{i_{j}} p_{i_{j}}$, where $\left\{p_{i_{j}}\right\}_{j=1}^{m^{\prime}}$ is the subset of $\left\{p_{i}\right\}_{i=1}^{m}$ of all those directed paths originating at $v$ and terminating at $w$. If $X=\left(X_{v}, \alpha(a)\right)$ is a $k$-representation of $\Gamma$ and $p$ is a directed path, we let $p(X)=\alpha\left(a_{1}\right) \circ \cdots \circ \alpha\left(a_{n}\right)$, where $p$ is the directed path $a_{1} \circ \cdots \circ a_{n}, a_{1}, \ldots, a_{n}$ arrows in $\Gamma$. If $t=\Sigma \mu_{i} p_{i}$ is a $k$-linear combination of directed paths in $\Gamma$ and $v, w \in V(\Gamma)$, we set

$$
c_{v, w}(t)(X)=\sum_{j=1}^{m^{\prime}} \mu_{i_{j}} p_{i_{j}}(X) \quad \text { where } c_{v, w}(t)=\sum_{j=1}^{m^{\prime}} \mu_{i_{j}} p_{i_{j}} .
$$

Note that the linear combination of the linear maps $\sum_{j=1}^{m^{\prime}}{ }_{i_{j}} p_{i_{j}}(X)$ makes sense since each $p_{i}(X)$ is a linear map from $X_{v}$ to $X_{w}$.

Let $\mathcal{G}$ be an index set. Let $\rho=\left\{t_{i}\right\}_{i \in g}$ be a set of $k$-linear combinations of directed paths in $\Gamma$. Let $X=\left(X_{v}, \alpha(a)\right)$ be a $k$-representation of $\Gamma$. We say $X$ satisfies the relations $\rho$ if for each $v, w \in V(\Gamma)$ and each $i \in \mathcal{G}, t_{i} \in \rho, c_{v, w}\left(t_{i}\right)(X)=0$.

We let $\operatorname{rep}(\Gamma, \rho)$ denote the full subcategory of $k$-representations of $\Gamma$ satisfying the relations $\rho$. We now state a fundamental theorem. 
THEOREM 1.1. Let $\Gamma$ be a finite directed graph and $\rho$ a set of relations on $\Gamma$. There is an equivalence of categories $E: \operatorname{rep}(\Gamma) \rightarrow \bmod (T(\Gamma))$, the category of finite-dimensional $T(\Gamma)$-modules, such that $E$ restricts to an equivalence of categories $E: \operatorname{rep}(\Gamma, \rho)$ $\rightarrow \bmod (T(\Gamma) / I)$, where $I$ is the ideal in $T(\Gamma)$ generated by the elements of $\rho$.

For a prcosf see $[\mathbf{5 , 6 ]}$. There is a similar result if $\Gamma$ is not a finite graph, but then one must give a slightly different definition of $T(\Gamma)$. We omit this since we have no need of it. The remainder of this paper shows how covering spaces of a quiver of $\Gamma$ are related to various gradings of $\Lambda$ and the categories of graded $\Lambda$-modules.

The next result will be needed in $\$ 3$ and indicates the importance of the components of a $k$-linear combination of directed paths.

Proposition 1.2. Let $\Gamma$ be a finite directed graph. Let I be an ideal in the free path algebra $T(\Gamma)$. Let $t=\sum_{i=1}^{n} \mu_{i} p_{i}$ be a finite $k$-linear combination of directed paths $p_{i}$ in $\Gamma$. Then $t \in I$ if and only if for all vertices $v, w$ in $\Gamma$ the $(v, w)$-component of $t, c_{v, w}(t)$, is an element of $I$.

Proof. To each vertex $v$ of $\Gamma$ there is an idempotent, $e_{v}$, in $T(\Gamma)$ corresponding to the path of length 0 at $v$. Since $1=\sum_{v \in V(\Gamma)} e_{v}$ and the $e_{v}$ 's are mutually orthogonal, we get that $t \in I \Leftrightarrow e_{v} t e_{w} \in I$ for all $v, w \in V(\Gamma)$. The result follows once we note that $e_{v} t e_{w}=c_{v, w}(t)$.

The above result shows that if we are concerned with $I$ we may assume that the elements of $\rho$ consist of linear combinations of paths with the same originating vertex and terminating vertex. Thus the reader may add this extra hypothesis to the definition of relations if he desires. If so, care must be taken in lifting relations (see §) for then, in general, relations will not lift to relations.

2. The coverings of graphs with relations. In this section we introduce the category of coverings of a graph with relations. We show that there is a universal object covering a finite directed graph with relations. In general, the graphs we consider in this section need not be finite. We will assume though that all graphs are path connected in that given any two vertices there is a (nondirected) path from one vertex to the other of finite length. We also assume all graphs are locally finite; that is, given a vertex there are at most a finite number of arrows entering and leaving that vertex.

Let $\Gamma$ be a directed graph. When viewing $\Gamma$ as a topological space we assume that we have a fixed embedding of $\Gamma$ in real 3-space, and that, with this embedding, $\Gamma$ satisfies the axioms of a graph given in [8, Chapter 6, §2]. Let $k$ be a fixed field. Recall, if $u, v \in V(\Gamma)$, the $(u, v)$-component of $t, c_{u, v}(t)=\sum_{j=1}^{l} \mu_{i_{j}} p_{i_{j}}$, where $\left\{p_{i_{j}}\right\}_{j=1}^{l}$ is the subset of the set of paths $\left\{p_{i}\right\}_{i=1}^{m}$ which originate at $u$ and terminate at $v$.

Now let $F: \Gamma_{1} \rightarrow \Gamma_{2}$ be a covering projection of graphs (in the topological sense-see [8, Chapters 5,6]). If $p$ is a directed path in $\Gamma_{1}$ then $F(p)$ is a directed path in $\Gamma_{2}$. If $t=\Sigma \mu_{i} p_{i}$ is a $k$-linear combination of directed paths in $\Gamma_{1}$, we let $F(t)=\Sigma \mu_{i} F\left(p_{i}\right)$, which is a $k$-linear combination of directed paths in $\Gamma_{2}$.

Next let $L: V\left(\Gamma_{2}\right) \rightarrow V\left(\Gamma_{1}\right)$ such that $L(v) \in F^{-1}(v)$. We call $L$ a lifting. By uniqueness of path lifting, if $p$ is a path (possibly nondirected) with origin $v$ and terminus $w$ in $\Gamma_{2}$, then we denote by $L(p)$ the unique path in $\Gamma_{1}$ originating at $L(v)$ 
such that $F(L(p))=p$. Note that $L(p)$ in general does not terminate at $L(w)$. If $t=\sum_{i=1}^{m} \mu_{i} p_{i}$ is a $k$-linear combination of directed paths in $\Gamma_{2}$, we let $L(t)$ denote $\sum_{i=1}^{m} \mu_{i} L\left(p_{i}\right)$.

Finally if $F: \Gamma_{1} \rightarrow \Gamma_{2}$ is a covering and $v_{1} \in V\left(\Gamma_{1}\right)$, we let $\pi_{1}\left(\Gamma_{1}, v_{1}\right)$ be the fundamental group of $\Gamma_{1}$ and $F_{*}: \pi_{1}\left(\Gamma_{1}, v_{1}\right) \rightarrow \pi_{1}\left(\Gamma_{2}, F\left(v_{1}\right)\right)$ be the map induced by $F$ (see [8, Chapter 2]). We say $F: \Gamma_{1} \rightarrow \Gamma_{2}$ is a regular covering if $F_{*}\left(\pi_{1}\left(\Gamma_{1}, v_{1}\right)\right)$ is a normal subgroup of $\pi_{1}\left(\Gamma_{2}, F\left(v_{1}\right)\right)$. If $r$ is a closed path in $\Gamma_{1}$ originating at $v_{1}$ we may use the notation $[r] \in \pi_{1}\left(\Gamma_{1}, v_{1}\right)$ to denote the class in $\pi_{1}\left(\Gamma_{1}, v_{1}\right)$ that contains $r$.

We say $(\Gamma, \rho)$ is a graph with relations if $\Gamma$ is a graph and $\rho$ is a set of $k$-linear combinations of directed paths in $\Gamma$. If $\left(\Gamma_{1}, \rho_{1}\right)$ and $\left(\Gamma_{2}, \rho_{2}\right)$ are graphs with relations, we say $F:\left(\Gamma_{1}, \rho_{1}\right) \rightarrow\left(\Gamma_{2}, \rho_{2}\right)$ is a morphism of graphs with relations if $F$ : $\Gamma_{1} \rightarrow \Gamma_{2}$ is a regular covering projection of graphs satisfying:

(A) $\rho_{1}=\left\{L(t): L: V\left(\Gamma_{2}\right) \rightarrow V\left(\Gamma_{1}\right)\right.$ is a lifting and $\left.t \in \rho_{2}\right\}$;

(B) if $t \in \rho_{1}$ and $u, v \in V\left(\Gamma_{2}\right)$ then there exist $u^{\prime}, v^{\prime} \in V\left(\Gamma_{1}\right)$ such that $F\left(c_{u^{\prime}, v^{\prime}}(t)\right)$ $=c_{u, v}(F(t))$.

Proposition 2.1. Let $F:\left(L_{1}, \rho_{1}\right) \rightarrow\left(L_{2}, \rho_{2}\right)$ be a morphism of graphs with relations. Then:

(i) $\rho_{1}$ determines $\rho_{2}$;

(ii) $\rho_{2}$ determines $\rho_{1}$;

(iii) let $t=\sum_{i=1}^{n} \mu_{i} p_{i} \in \rho_{2}$ and $p_{1}, p_{2}$ are both paths in the same component of $t$; that is, $p_{1}$ and $p_{2}$ have the same origin and terminus. Let $L$ be a lifting. Then $L\left(p_{1}\right)$ and $L\left(p_{2}\right)$ have the same origin and terminus in $\Gamma_{2}$.

Proof. By (A), $\rho_{2}=\left\{F(t) \mid t \in \rho_{1}\right\}$ and hence (i) follows. (ii) also immediately follows from (A). (iii) is a consequence of $(A)$ and $(B)$.

We turn to the question: given a regular covering $F: \Gamma_{1} \rightarrow \Gamma_{2}$ and a set of relations $\rho_{2}$ on $\Gamma_{2}$, under what circumstances can a set of relations $\rho_{1}$ on $\Gamma_{1}$ be defined so that $F:\left(\Gamma_{1}, \rho_{1}\right) \rightarrow\left(\Gamma_{2}, \rho_{2}\right)$ is a morphism of graphs with relations? To answer this question, we define a symmetric relation, denoted by $\sim$, on the set of directed paths of a graph with relations. Let $(\Gamma, \rho)$ be a graph with relations. We say two directed paths $p$ and $q$ in $\Gamma$ are related, $p \sim q$, if there is $t \in \rho$ and vertices $v, w \in V(\Gamma)$ such that

$$
c_{v, u}(t)=\mu p+\mu^{\prime} q+\sum_{j=1}^{m} \mu_{j} q_{j},
$$

where $\mu, \mu^{\prime}$ are nonzero elements of $k, \mu_{j} \in k, q_{j}$ are directed paths in $\Gamma$ distinct from $p$ and $q, j=1, \ldots, m$. Note that $\sim$ is a symmetric relation.

LEMMA 2.2. If $F:\left(\Gamma_{1}, \rho_{1}\right) \rightarrow\left(\Gamma_{2}, \rho_{2}\right)$ is a morphism of graphs with relations then:

(i) if $p \sim q$ in $\Gamma_{1}$ then $F(p) \sim F(q)$ in $\Gamma_{2}$;

(ii) if $p \sim q$ in $\Gamma_{2}$ and $L$ is a lifting then $L(p) \sim L(q)$ in $\Gamma_{1}$.

Proof. (i) follows immediately from (A) and (ii) follows from Proposition 2.1(iii).

Let $(\Gamma, \rho)$ be a graph with relations. Let $v \in V(\Gamma)$. We define the relation subgroup of $(\Gamma, \rho)$, denoted $N(\Gamma, \rho, v)$, to be the normal subgroup of $\pi_{1}(\Gamma, v)$ 
generated by elements of the form $\left[\gamma^{-1} p^{-1} q \gamma\right]$, where $p \sim q$ and $\gamma$ is a (nondirected) path from $v$ to the origin of $q$, and $p^{-1}$ denotes the inverse path from the terminus of $p$ to the origin of $p$ along $p$. As an immediate consequence of Lemma 2.2 we get

Corollary 2.3. Let $F:\left(\Gamma_{1}, \rho_{1}\right) \rightarrow\left(\Gamma_{2}, \rho_{2}\right)$ be a morphism of graphs with relations and let $v_{1} \in V\left(\Gamma_{1}\right)$ and $v_{2}=F\left(v_{1}\right)$. Then $F_{*}\left(N\left(\Gamma_{1}, \rho_{1}, v_{1}\right)\right)=N\left(\Gamma_{2}, \rho_{2}, v_{2}\right)$.

The next result answers the question asked earlier.

THEOREM 2.4. Let $F: \Gamma_{1} \rightarrow \Gamma_{2}$ be a regular covering of graphs. Let $v_{1} \in V\left(\Gamma_{1}\right)$ and $v_{2}=F\left(v_{1}\right)$. If $\rho_{2}$ is a set of relations in $\Gamma_{2}$ then there exists a unique set of relations $\rho_{1}$ in $\Gamma_{1}$ such that $F:\left(\Gamma_{1}, \rho_{1}\right) \rightarrow\left(\Gamma_{2}, \rho_{2}\right)$ is a morphism of graphs with relations if and only if $F_{*}\left(\pi_{1}\left(\Gamma_{1}, v_{1}\right)\right)$ contains $N\left(\Gamma_{2}, \rho_{2}, v_{2}\right)$.

Proof. If $\rho_{1}$ exists it is unique by Proposition 2.1. Furthermore, if $\rho_{1}$ exists then, by Corollary 2.3, $F_{*}\left(N\left(\Gamma_{1}, \rho_{1}, v_{1}\right)\right)=N\left(\Gamma_{2}, \rho_{2}, v_{2}\right)$ and we conclude that $F_{*}\left(\pi_{1}\left(\Gamma_{1}, v_{1}\right)\right)$ contains $N\left(\Gamma_{2}, \rho_{2}, v_{2}\right)$. Now suppose $F_{*}\left(\pi_{1}\left(\Gamma_{1}, v_{1}\right)\right)$ contains $N\left(\Gamma_{2}, \rho_{2}, v_{2}\right)$. Define $\rho_{1}=\left\{\sum \mu_{i} L\left(p_{i}\right): L\right.$ is a lifting and $\left.\Sigma \mu_{i} p_{i} \in \rho_{2}\right\}$. To show $F$ : $\left(\Gamma_{1}, \rho_{1}\right) \rightarrow\left(\Gamma_{2}, \rho_{2}\right)$ is a morphism of graphs with relations, we must verify $(\mathrm{A})$ and (B). By definition, (A) follows. Let $t=\Sigma \mu_{i} p_{i} \in \rho_{2}$ and let $L$ be a lifting. Let $v, w \in V\left(\Gamma_{2}\right)$. We must show there exist $v^{*}, w^{*} \in V\left(\Gamma_{1}\right)$ such that $c_{v, w}(t)=$ $F\left(c_{v^{*}, w^{*}}(L(t))\right)$. Suppose $c_{v, w}(t)=\sum_{j=1}^{m} \nu_{j} q_{j}$. Thus each $q_{j}$ is a directed path in $\Gamma_{2}$ from $v$ to $w$. Let $v^{*}=L(v)$. Let $w_{j}$ be the terminating vertex of $L\left(q_{j}\right)$. We must show $w_{1}=w_{2}=\cdots=w_{m}$. If so, $c_{v^{*}, w_{1}}(L(t))=L\left(c_{v, w}(t)\right)$ and (B) follows. Fix $j$, $1 \leqslant j \leqslant m$. Let $\gamma^{*}$ be a (nondirected) path in $\Gamma_{1}$ from $v_{1}$ to $L(v)=v^{*}$. Let $\gamma=F\left(\gamma^{*}\right)$. Then $\gamma$ is a (nondirected) path in $\Gamma_{2}$ from $v_{2}$ to $v$. Now $\left[\gamma^{-1} q_{j}^{-1} q_{1} \gamma\right] \in$ $N\left(\Gamma_{2}, \rho_{2}, v_{2}\right)$. Thus $\left[\gamma^{-1} q_{j}^{-1} \gamma\right] \in F_{*}\left(\pi_{1}\left(\Gamma_{1}, v_{1}\right)\right)$. By standard results in the theory of covering spaces (e.g. by [8, Chapter $5, \S 8])$, it follows that $\gamma^{*-1} \tilde{q}_{j}^{-1} L\left(q_{1}\right) \gamma^{*}$ is a closed path in $\Gamma_{1}$, where $\tilde{q}_{j}$ is the unique path in $\Gamma_{1}$ with terminus $w_{1}$ lifting $q_{j}$. But then the origin of $\tilde{q}_{j}$ must be $v^{*}=L(v)$ and, hence, $\tilde{q}_{j}=L\left(q_{j}\right)$, and we conclude that $w_{j}=w_{1}$. This completes the proof.

We can now show that universal coverings exist. More precisely, if $(\Gamma, \rho)$ is a graph with relations, let $\operatorname{Cov}(\Gamma, \rho)$ denote the category whose objects are $F$ : $\left(\Gamma^{\prime}, \rho^{\prime}\right) \rightarrow(\Gamma, \rho)$ such that $F$ is a morphism of graphs with relations. The morphisms in $\operatorname{Cov}(\Gamma, \rho)$ are morphisms

$$
H:\left(\left(\Gamma^{\prime}, \rho^{\prime}\right) \stackrel{F}{\rightarrow}(\Gamma, \rho)\right) \rightarrow\left(\left(\Gamma^{\prime \prime}, \rho^{\prime \prime}\right) \stackrel{F^{\prime}}{\rightarrow}(\Gamma, \rho)\right)
$$

where $H: \Gamma^{\prime} \rightarrow \Gamma^{\prime \prime}$ is a covering such that

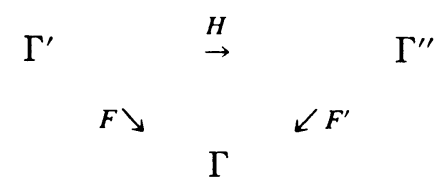

commutes. We denote elements of $\operatorname{Cov}(\Gamma, \rho)$ by $\left(\Gamma^{\prime}, \rho^{\prime}, F\right)$ and morphisms by $H$ : $\left(\Gamma^{\prime}, \rho^{\prime}, F\right) \rightarrow\left(\Gamma^{\prime \prime}, \rho^{\prime \prime}, F^{\prime}\right)$.

Lemma 2.5. If $H:\left(\Gamma^{\prime}, \rho^{\prime}, F\right) \rightarrow\left(\Gamma^{\prime \prime}, \rho^{\prime \prime}, F^{\prime}\right)$ is a morphism $\operatorname{Cov}(\Gamma, \rho)$ then $H$ : $\left(\Gamma^{\prime}, \rho^{\prime}\right) \rightarrow\left(\Gamma^{\prime \prime}, \rho^{\prime \prime}\right)$ is a morphism of graphs with relations. 
Proof. Let $v^{\prime} \in \Gamma^{\prime}, v^{\prime \prime} \in \Gamma^{\prime \prime}$ and $v \in \Gamma$ such that $F\left(v^{\prime}\right)=v, F^{\prime}\left(v^{\prime \prime}\right)=v$ and $H\left(v^{\prime}\right)=v^{\prime \prime}$. Each map $H_{*}: \pi_{1}\left(\Gamma^{\prime}, v^{\prime}\right) \rightarrow \pi_{1}\left(\Gamma^{\prime \prime}, v^{\prime \prime}\right), F_{*}: \pi_{1}\left(\Gamma^{\prime}, v^{\prime}\right) \rightarrow \pi_{1}(\Gamma, v)$ and $F_{*}^{\prime}: \pi_{1}\left(\Gamma^{\prime \prime}, v^{\prime \prime}\right) \rightarrow \pi_{1}(\Gamma, v)$ is a morphism [8, Theorem 4.1] and $F_{*}^{\prime} H_{*}=F_{*}$. Thus $F_{*}^{\prime} H_{*}\left(\pi_{1}\left(\Gamma^{\prime}, v^{\prime}\right)\right)$ is a normal subgroup of $\pi_{1}(\Gamma, v)$ and

$$
F_{*}^{\prime} H_{*}\left(\pi_{1}\left(\Gamma^{\prime}, v^{\prime}\right)\right) \subseteq F_{*}^{\prime}\left(\pi_{1}\left(\Gamma^{\prime \prime}, v^{\prime \prime}\right)\right) \subseteq \pi_{1}(\Gamma, v)
$$

Hence we conclude $H_{*}\left(\pi_{1}\left(\Gamma^{\prime}, v^{\prime}\right)\right)$ is a normal subgroup of $\pi_{1}\left(\Gamma^{\prime \prime}, v^{\prime \prime}\right)$ and $H$ : $\Gamma^{\prime} \rightarrow \Gamma^{\prime \prime}$ is a regular covering. Next, since

$$
F_{*}\left(N\left(\Gamma^{\prime}, \rho^{\prime}, v^{\prime}\right)\right)=N(\Gamma, \rho, v)=F_{*}^{\prime}\left(N\left(\Gamma^{\prime \prime}, \rho^{\prime \prime}, v^{\prime \prime}\right)\right),
$$

we conclude that $N\left(\Gamma^{\prime \prime}, \rho^{\prime \prime}, v^{\prime \prime}\right)$ is contained in $H_{*}\left(\pi_{1}\left(\Gamma^{\prime}, v^{\prime}\right)\right)$. By Theorem 2.4 there is a set of relations $\tilde{\rho}$ in $\Gamma^{\prime}$ such that $H:\left(\Gamma^{\prime}, \tilde{\rho}\right) \rightarrow\left(\Gamma^{\prime \prime}, \rho^{\prime \prime}\right)$ is a morphism of graphs with relations. But then $F^{\prime} \circ H:\left(\Gamma^{\prime}, \tilde{\rho}\right) \rightarrow(\Gamma, \rho)$ is a morphism of graphs with relations. Since $F^{\prime} \circ H=F$, by unicity of relations 2.2 we conclude $\rho^{\prime}=\tilde{\rho}$ and we are done.

The next result gives the existence and uniqueness of universal objects in $\operatorname{Cov}(\Gamma, \rho)$.

THEOREM 2.6. Let $(\Gamma, \rho)$ be a graph with relations. There is an object $F^{*}$ : $\left(\Gamma^{*}, \rho^{*}\right) \rightarrow(\Gamma, \rho)$ in $\operatorname{Cov}(\Gamma, \rho)$ such that for every $F^{\prime}:\left(\Gamma^{\prime}, \rho\right) \rightarrow(\Gamma, \rho)$ in $\operatorname{Cov}(\Gamma, \rho)$ there is a morphism $H:\left(\Gamma^{*}, \rho^{*}, F^{*}\right) \rightarrow\left(\Gamma^{\prime}, \rho^{\prime}, F\right)$ in $\operatorname{Cov}(\Gamma, \rho)$. Moreover, given $v \in V(\Gamma), v^{\prime} \in V\left(\Gamma^{\prime}\right)$ and $v^{*} \in V\left(\Gamma^{*}\right)$ such that $F^{*}\left(v^{*}\right)=v=F^{\prime}\left(v^{\prime}\right)$, then $H$ can be uniquely chosen so that $H\left(v^{*}\right)=v^{\prime}$. Finally $\left(\Gamma^{*}, \rho^{*}, F^{*}\right)$ is unique up to isomorphism in $\operatorname{Cov}(\Gamma, \rho)$.

Proof. By the theory of covering spaces, there is a covering, $F^{*}: \Gamma^{*} \rightarrow \Gamma$, such that if $v^{*} \in V\left(\Gamma^{*}\right)$ then $\left(F^{*}\right)_{*}\left(\pi_{1}\left(\Gamma^{*}, v^{*}\right)\right)=N\left(\Gamma, \rho, F^{*}\left(v^{*}\right)\right)$. Since $N\left(\Gamma, \rho, F^{*}\left(v^{*}\right)\right)$ is a normal subgroup of $\pi_{1}\left(\Gamma, \rho, F^{*}\left(v^{*}\right)\right)$, we conclude $F^{*}: \Gamma^{*} \rightarrow \Gamma$ is unique up to isomorphism and is a regular covering. By Theorem 2.4 there is a unique set of relations $\rho^{*}$ on $\Gamma^{*}$ such that $F^{*}:\left(\Gamma^{*}, \rho^{*}\right) \rightarrow(\Gamma, \rho)$ is a morphism of graphs with relations and hence an object in $\operatorname{Cov}(\Gamma, \rho)$. Now let $F^{\prime}:\left(\Gamma^{\prime}, \rho^{\prime}\right) \rightarrow(\Gamma, \rho)$ be an object in $\operatorname{Cov}(\Gamma, \rho)$. Choose $v \in V(\Gamma), v^{\prime} \in V\left(\Gamma^{\prime}\right), v \in V(\Gamma)$ such that $F^{*}\left(v^{*}\right)=$ $F^{\prime}\left(v^{\prime}\right)$. Then, since

$$
\left(F^{\prime}\right)_{*}\left(\pi_{1}\left(\Gamma^{\prime}, v^{\prime}\right)\right) \supseteq N(\Gamma, \rho, v)=\left(F^{*}\right)_{*}\left(\rho_{1}\left(\Gamma^{*}, v^{*}\right)\right),
$$

there is a unique covering $H: \Gamma^{*} \rightarrow \Gamma^{\prime}$ such that $F^{\prime} \circ H=F^{*}$. This completes the proof.

We end this section by studying $k$-representations of graphs with relations (see $\S 1$ for definitions) with respect to morphisms of graphs with relations. We get the following useful result.

Proposition 2.7. Let $F:\left(\Gamma_{1}, \rho_{1}\right) \rightarrow\left(\Gamma_{2}, \rho_{2}\right)$ be a morphism of graphs with relations. Then $F$ induces an exact additive functor $F: \operatorname{rep}\left(\Gamma_{1}, \rho_{1}\right) \rightarrow \operatorname{rep}\left(\Gamma_{2}, \rho_{2}\right)$.

Proof. Let $X=\left(X_{v}, \alpha(a)\right)$ be a $k$-representation of $\left(\Gamma_{1}, \rho_{1}\right)$. Define $F(X)=$ $\left(Y_{w^{\prime}}, \beta(b)\right)$ as follows: if $w \in V\left(\Gamma_{2}\right)$ then $Y_{w^{\prime}}=\amalg_{v \in F^{-1}(w)} X_{v}$ as vector spaces. Let $b \in A\left(\Gamma_{2}\right)$ be an arrow from $w$ to $w^{\prime}$. Note that if $a \in F^{-1}(b), a$ is an arrow in $\Gamma_{1}$ from $v$ to $v^{\prime}$ for some $v \in F^{-1}(w)$ and $v^{\prime} \in F^{-1}\left(v^{\prime}\right)$. Thus $\alpha(a): X_{v} \rightarrow X_{v^{\prime}}$ induces a map also denoted $\alpha(a): Y_{u^{\prime}} \rightarrow Y_{u^{\prime}}$. Define $\beta(b)=\amalg_{a \in F^{-1}(b)} \alpha(a): Y_{u^{\prime}} \rightarrow Y_{u^{\prime}}$. Next, it 
follows directly from the definitions and properties (A), (B) that if $\left(X_{v}, \alpha(a)\right)$ satisfies the relations $\rho_{1}$, then $F\left(X_{v}, \alpha(a)\right)$ satisfies the relations $\rho_{2}$. Finally if

$$
B=\left(B_{v}\right):\left(X_{v}, \alpha(a)\right) \rightarrow\left(X_{v}^{\prime}, \alpha^{\prime}(a)\right)
$$

is a morphism in $\operatorname{rep}\left(\Gamma_{1}, \rho_{1}\right)$, we define $F(B): F\left(X_{v}, \alpha(a)\right) \rightarrow\left(X_{v}^{\prime}, \alpha^{\prime}(a)\right)$ by $F(B)_{n}$ : $\mathrm{II}_{v \in F^{-1}(w)} X_{v} \rightarrow \amalg_{v \in F^{-1}(w)} X_{v}^{\prime}$ is given by $F(B)_{w}=\amalg_{v \in F^{-1}(w)} B_{v}$. Again it is not difficult to verify that we get a functor $F: \operatorname{rep}\left(\Gamma_{1}, \rho_{1}\right) \rightarrow \operatorname{rep}\left(\Gamma_{2}, \rho_{2}\right)$. The functor $F$ is clearly exact and additive.

In the next section we show that $F$ can be reinterpreted as a forgetful functor.

3. Group-graded algebras. Let $\Lambda$ be a $k$-algebra. Let $G$ be a group with identity element $e$. We say $\Lambda=\amalg_{g \in G} \Lambda_{g}$ is a $G$-grading of $\Lambda$ if the sum is direct as $k$-vector spaces under addition and if for all $g, h \in G, \Lambda_{g} \Lambda_{h} \subseteq \Lambda_{g h}$. We say a group grading is full if $\left\{g \in G: \Lambda_{g} \neq 0\right\}$ generates $G$ as a group. Note that if $\Lambda=\amalg \Lambda_{g}$ is not full, letting $G^{\prime}$ be the subgroup generated by $\left\{g: \Lambda_{g} \neq 0\right\}$, then $\Lambda=\amalg \Lambda_{g}$ can be considered a full $G^{\prime}$-grading of $\Lambda$.

Let $\Lambda=\amalg \Lambda_{g}$ be a $G$-graded ring. We let $\operatorname{gr}_{G}(\Lambda)$ (or just $\operatorname{gr}(\Lambda)$ ) denote the category whose objects are finite-dimensional $G$-graded $\Lambda$-modules $M=\amalg_{g \in G} M_{g}$ (where $\Lambda_{h} M_{g} \subseteq M_{h g}$ ) and whose morphisms are $\Lambda$-module maps $f: \amalg M_{g} \rightarrow \amalg N_{g}$, so that for all $g \in G, f\left(M_{g}\right) \subseteq N_{g}$. We call such maps degree e maps. If $\amalg M_{g}$, $\amalg N_{g}$ are objects in $\operatorname{gr}(\Lambda)$ and $f: \amalg M_{g} \rightarrow \amalg N_{g}$ is a $\Lambda$-module map such that there is an $h \in G$ such that $f\left(M_{g}\right) \subseteq N_{g h}$, we say $f$ is a degree $h$ map. The following result is proved (e.g. see [3]) for $\mathbf{Z}$-graded rings, where $\mathbf{Z}$ denotes the group of integers.

LEMMA 3.1. If $\Lambda=\amalg_{g \in G} \Lambda_{g}$ is a G-graded ring and $M=\amalg_{g \in G} M_{g}$ and $N=\amalg_{g \in G} N_{g}$ are finite-dimensional $G$-graded $\Lambda$-modules, then every $\Lambda$-module map $f: M \rightarrow N$ can be uniquely written as a sum $\Sigma_{g \in G} f_{g}$ where $f_{g}$ is a degree $g$ map.

Let $\phi: \operatorname{gr}(\Lambda) \rightarrow \bmod (\Lambda)$ denote the forgetful functor, where $\bmod (\Lambda)$ is the category of finite-dimensional $\Lambda$-modules. If $M$ is a finite-dimensional $\Lambda$-module we say $M$ is gradable if there exists an object $\amalg_{g \in G} X_{g}$ in $\operatorname{gr}(\Lambda)$ such that $\phi\left(\amalg_{g \in G} X_{g}\right)=$ M.

Before giving the main theorems we need to introduce a special type of grading which plays a central role in what follows. Let $\Gamma$ be a finite directed graph and let $T$ denote the free path $k$-algebra associated to $\Gamma$. Let $G$ be a group. Let $W: A(\Gamma) \rightarrow G$ be a set map. We call $W$ a weight function. Then $W$ induces a $G$-grading on $T$ as follows: if $p$ is a directed path in $\Gamma$ and $p=a_{1} \cdots a_{n}$, where $a_{i} \in A(\Gamma)$, then we set $W(p)=W\left(a_{1}\right) \cdots W\left(a_{n}\right) \in G$. Let $T_{g}=\left\{\sum_{i=1}^{n} \mu_{i} p_{i}: \mu_{i} \in k, p_{i}\right.$ is a directed path in $\Gamma$ with $W\left(p_{i}\right)=g$ \}. It is immediate that $T=\amalg_{g \in G} T_{g}$ is a $G$-grading of $T$. We call this the grading induced by (the weight function) $W$. Clearly the grading induced by $W$ is full if and only if $\{W(a) \in G: a \in A(\Gamma)\}$ generates $G$. Let $I$ be an ideal in $T$ and $\Lambda=T / I$. If $I$ is a homogeneous ideal in $T$ then $T=\amalg T_{g}$ induces a $G$-grading of $\Lambda$, also called the grading induced by $W$.

If $W: A(\Gamma) \rightarrow G$ is a weight function and $p=a_{1}^{e_{1}} \cdots a_{n}^{e_{n}}$ is a nondirected path in $\Gamma$, where $a_{i} \in A(\Gamma)$ and $e_{i}= \pm 1, i=1, \ldots, n$, then we define $W(p)=W\left(a_{1}\right)^{e_{1}}$ $\cdots W\left(a_{n}\right)^{e_{n}} \in G$. We say the $G$-grading induced by $W$ is connected if for all 
$v, w \in V(\Gamma)$ and for all $g \in G$ there is a (possibly nondirected) path $p$ from $v$ to $w$ such that $W(p)=g$. Note that assuming $I$ contains no path of length 1 (i.e., arrow), then if the induced $G$-grading on $\Lambda$ is connected, then the $G$-grading is full.

We now state our first main theorem.

THEOREM 3.2. Let $\Lambda$ be a finitely generated indecomposable k-algebra. Let $\Gamma_{0}$ be a connected finite directed graph and $\rho_{0}$ a set of relations on $\Gamma_{0}$ so that $T\left(\Gamma_{0}\right) / I$ is isomorphic to $\Lambda$, where $T\left(\Gamma_{0}\right)$ is the free path $k$-algebra associated to $\Gamma_{0}$ and $I$ is the ideal in $T\left(\Gamma_{0}\right)$ generated by the elements of $\rho_{0}$.

Let $F:(\Gamma, \rho) \rightarrow\left(\Gamma_{0}, \rho_{0}\right)$ be an object in $\operatorname{Cov}\left(\Gamma_{0}, \rho_{0}\right)$. Let $G$ be the group of automorphisms of the regular covering $F: \Gamma \rightarrow \Gamma_{0}$. Then there is a weight function $W$ : $A(\Gamma) \rightarrow G$ such that $\Lambda$ may be given the G-grading induced by $W$. Giving $\Lambda$ the $G$-grading induced by $W$, there is a natural equivalence, $E^{*}$, of categories between $\operatorname{gr}(\Lambda)$ and $\operatorname{rep}(\Gamma, \rho)$ such that the following diagram commutes:

$$
\begin{array}{ccc}
\operatorname{rep}(\Gamma, \rho) & \stackrel{E^{*}}{\approx} & \operatorname{gr}(\Lambda) \\
F \downarrow & & \downarrow \phi \\
\operatorname{rep}\left(\Gamma_{0}, \rho_{0}\right) & \stackrel{E}{\approx} & \bmod (\Lambda)
\end{array}
$$

where $F$ is defined in 2.7, and $E$ is the functor of 1.1 .

Proof. We identify $\Lambda$ and $T\left(\Gamma_{0}\right) / I$. Let $T=T\left(\Gamma_{0}\right)$. Consider the automorphism group $G$ of the regular covering $F: \Gamma \rightarrow \Gamma_{0}$. Then $G$ acts on $V(\Gamma)$ and we denote this action by $v^{g}$ if $v \in V(\Gamma)$ and $g \in G$. Thus, $\left(v^{g}\right)^{h}=v^{g h}$ for $g, h \in G$. Let $L$ : $V\left(\Gamma_{0}\right) \rightarrow V(\Gamma)$ be a lifting. We define a weight function $W: A\left(\Gamma_{0}\right) \rightarrow G$ as follows: let $a \in A\left(\Gamma_{0}\right)$ be an arrow from vertex $v$ to vertex $w$. Set $W(a)=g$ if $L(A)$ is an arrow from $L(v)$ to $L(w)^{g}$. First note that since the action of each nonidentity element of $G$ on $\Gamma$ has no fixed point, the weight of an arrow is well defined. Next note that $F^{-1}(v)=\left\{L(v)^{g}: g \in G\right\}$ since $F: \Gamma \rightarrow \Gamma_{0}$ is a regular covering, and we conclude each arrow in $A\left(\Gamma_{0}\right)$ has a weight. This defines a weight function and we get $T=\amalg_{g \in G} T_{g}$, the $G$-grading induced by $W$.

Now we show that a directed path $p$ from $v$ to $w$ has weight $g \Leftrightarrow L(p)$ is a path from $L(v)$ to $L(w)^{g}$. It suffices to show that if $p$ is a directed path from $v$ to $w$ of weight $g$ and $q$ is a directed path from $u$ to $v$ of weight $h$, then $L(p q)$ is a path from $L(u)$ to $L(w)^{g h}$. Now $L(q)$ is a directed path from $L(u)$ to $(L(v))^{h}$ and $L(p)$ is a path from $L(v)$ to $L(w)^{g}$. Letting $h$ act on $L(p)$, we get that $L(p)^{h}$ is a directed path from $L(v)^{h}$ to $\left(L(w)^{g}\right)^{h}=L(w)^{g h}$. By uniqueness of path lifting, $L(p)^{h} \circ L(q)=L(p q)$, which is a path from $L(u)$ to $L(w)^{g h}$. Hence, $p q \in T_{g h}$. Thus $T=\amalg_{g \in G} T_{g}$ is a $G$-grading on $T$.

Let $I$ be the ideal in $T$ generated by the elements of $\rho_{0}$. To show that the $G$-grading on $T$ induces a $G$-grading on $\Lambda$, we must show that $I$ is a homogeneous ideal in $T$. By 1.2 it suffices to show that if $t \in \rho_{0}$ and $v, w$ are vertices in $\Gamma_{0}$, then $c_{v, w}(t)$ is homogeneous; i.e., every path $p$ occurring in $c_{v, w}(t)$ has the same weight. Let $t \in \rho_{0}$ and $v, w \in V\left(\Gamma_{0}\right)$. Since each path occurring in $c_{v, w}(t)$ lifts to a path 
originating at $L(v)$, we must show each such path has the same terminal vertex. But this follows from Proposition 2.1(iii). Thus we conclude that $\Lambda$ is a $G$-graded factor ring of $T$.

Keeping $L$ fixed, we now construct an equivalence of categories $E^{*}: \operatorname{rep}(\Gamma, \rho) \rightarrow$ $\operatorname{gr}(\Lambda)$. Let $X=\left(X_{v}, \alpha(a)\right)$ be the $k$-representation of $\Gamma$ satisfying $\rho$. Let

$$
E^{*}(X)_{g}=\coprod_{v \in V\left(\Gamma_{0}\right)} X_{L(v)^{s}}, \quad E^{*}(X)=\coprod_{g \in G} E^{*}(X)_{g} .
$$

To define a $G$-graded $\Lambda$-action on $E^{*}(X)$ we need only define a graded $T$-action by describing the action of a directed path $p$ in $T$. Suppose the weight of $p$ is $g$ and $p$ is a path from $v$ to $w$ in $\Gamma_{0}$. Then we set $p X_{L(u)^{h}}=0$ unless $u=v$. If $x \in X_{L(v)^{h}}$ we define $p \cdot x$ as follows. We have that $L(p)$ is a path in $\Gamma$ from $L(v)$ to $L(w)^{g}$. Hence $L(p)^{h}$ is a directed path from $L(v)^{h}$ to $L(w)^{(g h)}$. Thus $L(p)^{h}(X)$ is a linear map from $X_{L(v)^{h}}$ to $X_{L(w)^{(g h)}}$ (see $\S 1$ for definition of $L(p)^{h}(X)$ ). Define $p \cdot x=$ $L(p)^{h}(X)(x) \in X_{L(w)^{(g h)}}$. It follows from this definition that $T_{g} \cdot E^{*}(X)_{h} \subseteq E^{*}(X)_{g h}$. Hence $E^{*}(X)=\amalg E^{*}(X)_{g}$ is a $G$-graded $T$-module. To show $I^{*}(X)=0$ we need only show $t E^{*}(X)=0$ for all $t \in \rho_{0}$. But

$$
t E^{*}(X)=0 \Leftrightarrow c_{u, v}(t) E^{*}(X)=0 \text { for all } u, v \in V\left(\Gamma_{0}\right)
$$

(see Proposition 1.2). Now let $t \in \rho_{0}, u, v \in V\left(\Gamma_{0}\right)$. Let $x \in E^{*}(X)$. If we show $c_{u, v}(t) x=0$ for all $x \in X_{L(w)^{h}}, w \in V\left(\Gamma_{0}\right), h \in G$, we will have our result. Thus assume $x \in X_{L(w)^{h}}$. Then $c_{u, v}(t) x=0$ if $w \neq u$. Thus we may assume $w=u$. But by Proposition 2.1(iii), there is some $g \in G$ such that $L\left(c_{u, v}(t)\right)=c_{L(u), L(v)^{g}}(L(t))$. But

$$
L(t)^{h} \in \rho \Leftrightarrow c_{L(u)^{h}, L(v)^{g^{h}}}\left(L(t)^{h}\right)(X)=0
$$

since $X$ satisfies the relations $\rho$ (see $\S 1)$. Thus we have shown that $E^{*}(X)$ is a $G$-graded $\Lambda$-module.

The definition of $E^{*}$ on the morphisms in $\operatorname{rep}(\Gamma, \rho)$ is easily given and left to the reader. The proof that $E^{*}$ is an equivalence of categories is completely analogous to the proof that $E: \operatorname{rep}\left(\Gamma_{0}, \rho_{0}\right) \rightarrow \bmod (\Lambda)$ is an equivalence found in $[5,6]$. Finally the commutativity of $(*)$ is a direct consequence of the definitions.

Before discussing the unicity of the grading constructed in the above proof, we show that the grading is connected.

Proposition 3.3. Let $\Lambda=\mathrm{I}_{g \in G} \Lambda_{g}$ be the G-grading of $\Lambda$ constructed in Theorem 3.2 from a covering $F:(\Gamma, \rho) \rightarrow\left(\Gamma_{0}, \rho_{0}\right)$ and lifting $L: V\left(\Gamma_{0}\right) \rightarrow V(\Gamma)$, where $\Gamma_{0}$ is as in Theorem 3.2. Then the G-grading of $\Lambda$ is connected.

Proof. Let $g \in G$. Let $v, w \in V(\Gamma)$. The graph $\Gamma$ is path connected since $\Gamma_{0}$ is. Thus there is a nondirected path $p$ in $\Gamma$ from $v$ to $w^{g}$. Then $p=a_{1}^{e_{1}} a_{2}^{e_{2}} \cdots a_{r}^{e_{r}}$, where $a_{1}, \ldots, a_{r} \in A(\Gamma)$ and $e_{i}= \pm 1$. Now $F\left(a_{i}\right) \in A\left(\Gamma_{0}\right)$ for $i=1, \ldots, r$. Let $g_{i}=$ weight of $F\left(a_{i}\right)$. Finally $F(p)=F\left(a_{1}\right)^{e_{1}} F\left(a_{2}\right)^{e_{2}} \cdots F\left(a_{r}\right)^{e_{r}}$. Since $L(F(p))=p$ is a path from $v$ to $w^{g}$, it follows that $g_{1}^{e_{1}} \cdots g_{r}^{e_{r}}=g$. Thus the grading is connected.

Our first remark about Theorem 3.2 is that the grading on $\Lambda$ constructed in the proof is dependent on the choice of lifting $L$-see Example 4.2. It can be shown, 
though, that the concept of "graded equivalence" introduced in [3] can be generalized to $G$-graded algebras and then the $G$-gradings on $\Lambda$ given by two different liftings are graded equivalent.

Next suppose $\rho_{0}^{\prime}$ is another choice of generators for the ideal $I$ in $T\left(\Gamma_{0}\right)$. It may happen that in the $G$-grading constructed in Theorem 3.2 using $\rho_{0}$ and the lifting $L$, there is an element $t^{\prime} \in \rho_{0}^{\prime}$ and vertices $v, w \in V\left(\Gamma_{0}\right)$ so $c_{v, w}\left(t^{\prime}\right)$ is not homogeneous. But in this case $N\left(\Gamma_{0}, \rho_{0}^{\prime}, v_{0}\right)$ is not contained in $F_{*}\left(\pi_{1}(\Gamma, \rho)\right)$ for every $v_{0} \in V\left(\Gamma_{0}\right)$. See Example 4.2.

Before stating the second main theorem, we look more closely at the finite-dimensional case. Let $\Lambda$ be a finite-dimensional $k$-algebra. We first note that in the $G$-grading of $\Lambda$ constructed in Theorem 3.2, assuming $\Gamma_{0}$ is the quiver of $\Lambda$, see [1] for a definition, the Jacobson radical $r$ of $\Lambda$ is a homogeneous ideal since $\underline{r}$ is generated by the images under $T\left(\Gamma_{0}\right) \rightarrow T\left(\Gamma_{0}\right) / I=\Lambda$ of length 1 paths in $T\left(\Gamma_{0}\right)$. Also simple $\Lambda$-modules are gradable since simple $\Lambda$-modules correspond to $k$-representations of $\Gamma_{0},\left(X_{v}, \alpha(a)\right)$ such that $X_{v}=0$ for all but one vertex $w$ and $X_{w}=k$, and $\alpha(a)=0$ for all $a$. Such $k$-representations are obviously in the image of $F$ : $\operatorname{rep}(\Gamma, \rho) \rightarrow \operatorname{rep}\left(\Gamma_{0}, \rho_{0}\right)$ and we conclude, using Theorem 3.2, that simple $\Lambda$-modules are gradable.

In another paper we prove a partial converse; namely that if $\Lambda=\amalg_{g \in G} \Lambda_{g}$ is a $G$-graded Artin algebra and $G$ is torsion-free, then the Jacobson radical of $\Lambda$ is a homogeneous ideal and simple $\Lambda$-modules are gradable. The result is easily seen to be false in general if $G$ has torsion. For example, let $k$ be a field of characteristic $p$ and $G$ a finite $p$-group. Then the group algebra $k G$ is a $G$-graded Artin $k$-algebra, but the radical is not a homogeneous ideal.

We now give the second main theorem, which is a converse to Theorem 3.2.

THEOREM 3.4. Let $\Gamma_{0}$ be a finite connected directed graph and let $T$ be the free path $k$-algebra associated to $\Gamma_{0}$. Let I be an ideal in $T$. Let $\Lambda=T / I$. Let $G$ be a group. Suppose $\Lambda=\amalg_{g \in G} \Lambda_{g}$ is the G-grading induced by a weight function $W: A\left(\Gamma_{0}\right) \rightarrow G$. Assume the induced $G$-grading by $W$ is connected. Then there is a set of generators $\rho_{0}$ of $I$ and an object $F:(\Gamma, \rho) \rightarrow\left(\Gamma_{0}, \rho_{0}\right)$ of $\operatorname{Cov}\left(\Gamma_{0}, \rho_{0}\right)$ such that: (i) the automorphism group of the covering $F: \Gamma \rightarrow \Gamma_{0}$ is isomorphic to $G$; (ii) there is a lifting L: $V\left(\Gamma_{0}\right) \rightarrow V(\Gamma)$ such that the induced G-grading on $\Lambda$ given by $F:(\Gamma, \rho) \rightarrow\left(\Gamma_{0}, \rho_{0}\right)$, constructed in Theorem 3.2, is the same as the G-grading on $\Lambda$ induced by $W$.

Proof. By definition, since $\Lambda=\amalg_{g \in G} \Lambda_{g}$ is the $G$-grading induced by $W, I$ is a homogeneous ideal in $T=\amalg_{g \in G} T_{g}$, where $\amalg_{g \in G} T_{g}$ is the $G$-grading induced by $W$. Let $\rho_{0}$ be a set of homogeneous generators of $I$. Thus if $\sum_{i=1}^{n} \mu_{i} p_{i} \in \rho_{0}$ it follows that $W\left(p_{1}\right)=W\left(p_{i}\right), i=1, \ldots, n$. We now define the graph $\Gamma$. Let $V(\Gamma)=\left\{v^{g}: v \in\right.$ $\left.V\left(\Gamma_{0}\right), g \in G\right\}$. Let $A(\Gamma)=\left\{b^{g}: b \in A\left(\Gamma_{0}\right), W(b)=h, g \in G\right.$ and $b^{g}$ is an arrow from $v^{g}$ to $w^{g h}$ where $b$ is an arrow from $v$ to $w$ \}. Define $F: \Gamma \rightarrow \Gamma_{0}$ by $F\left(b^{g}\right)=b$. It follows that $F\left(v^{g}\right)=v$ for all $v^{g} \in V(\Gamma)$. First we show $\Gamma$ is connected. But this is a direct consequence of the connectedness of the $G$-grading induced by $W$ and the fact that if $p$ is a nondirected path from $v$ to $w$ in $\Gamma_{0}$ of weight $h$, then for each $g \in G, p$ lifts to a unique nondirected path from $v^{g}$ to $w^{g h}$. From these considerations, it 
follows that $F: \Gamma \rightarrow \Gamma_{0}$ is a covering. By construction, it is clear that $F: \Gamma \rightarrow \Gamma_{0}$ is a regular covering; for, if $p$ is a nondirected closed path in $\Gamma_{0}$, then each lifting is closed if and only if $W(p)=e$. Next we show $F_{*} \pi_{1}\left(\Gamma, v^{e}\right)$ contains $N\left(\Gamma_{0}, \rho_{0}, v\right)$ for some $v \in V(\Gamma)$. But the elements of $\rho_{0}$ are homogeneous. Thus the relation on directed paths $p \sim q$ given in $\S 2$ has the property $p \sim q$ implies $W(p)=W(q)$. But then if $\gamma$ is a nondirected path in $\Gamma_{0}, W\left(\gamma^{-1} p^{-1} q \gamma\right)=e$. If $p \sim q$, then if we lift $p$ and $q$ to directed paths with the same origin, then the lifted paths have the same terminus since $W(p)=W(q)$. Furthermore, any lifting of $\gamma^{-1} p^{-1} q \gamma$ is a closed path since $W\left(\gamma^{-1} p^{-1} q \gamma\right)=e$. From the definition of $N\left(\Gamma_{0}, \rho_{0}, v\right)$ we conclude that $N\left(\Gamma_{0}, \rho_{0} v\right)$ is contained in $F_{*} \pi_{1}\left(\Gamma, v^{e}\right)$. Applying Theorem 2.4, we conclude that there is a set of relations $\rho$ in $\Gamma$ such that $F:(\Gamma, \rho) \rightarrow\left(\Gamma_{0}, \rho_{0}\right)$ is an object in $\operatorname{Cov}\left(\Gamma_{0}, \rho_{0}\right)$.

Next, it is clear that one may identify $G$ with the automorphism group of the covering $F: \Gamma \rightarrow \Gamma_{0}$. Finally, consider the lifting $L: V\left(\Gamma_{0}\right) \rightarrow V(\Gamma)$ given by $L(v)=$ $v^{e}$. Then from $L$ we may construct a weight function $W^{\prime}: A\left(\Gamma_{0}\right) \rightarrow G$ as in Theorem 3.2. But we immediately see that $W^{\prime}=W$, and the proof of the theorem is complete.

4. Concluding remarks and examples. Let $\Gamma_{0}$ be a finite directed graph and $\rho_{0}$ a set of relations on $\Gamma_{0}$. Let $T$ be the free path algebra associated to $\Gamma_{0}$. Let $I$ be the ideal in $T$ generated by $\rho_{0}$. Let $\Lambda=T / I$. Let $F^{*}:\left(\Gamma^{*}, \rho^{*}\right) \rightarrow\left(\Gamma_{0}, \rho_{0}\right)$ be the universal cover of $\left(\Gamma_{0}, \rho_{0}\right)$ in $\operatorname{Cov}\left(\Gamma_{0}, \rho_{0}\right)$ described in Theorem 2.6. By Theorem 3.2, if $G^{*}$ is the automorphism group of the covering $F^{*}: \Gamma^{*} \rightarrow \Gamma_{0}$ there is a $G^{*}$-grading on $\Lambda$ induced by a weight function $W^{*}: A\left(\Gamma_{0}\right) \rightarrow G^{*}$. The next theorem relates the representations of the "universal grading" to arbitrary group-gradings.

THEOREM 4.1. Let $\Lambda=\amalg_{g \in G} \Lambda_{g}$ be a full connected G-grading induced by a weight function $W: A\left(\Gamma_{0}\right) \rightarrow G$. Then the full subcategory of $\bmod (\Lambda)$ consisting of $G^{*}$-gradable $\Lambda$-modules is contained in the full subcategory of $\bmod (\Lambda)$ consisting of G-gradable $\Lambda$-modules.

Proof. Let $\mathbb{Q}^{*}$ (respectively $\mathscr{Q}$ ) denote the full subcategory of $\bmod (\Lambda)$ consisting of $G^{*}$-gradable (resp. $G$-gradable) $\Lambda$-modules. Thus $\mathbb{Q}^{*}$ (resp. $\mathbb{Q}$ ) consists of objects of $\bmod (\Lambda)$ of the form $\phi^{*}(X)(\operatorname{resp} . \phi(X))$, where $\phi^{*}: \operatorname{gr}_{G *}(\Lambda) \rightarrow \bmod (\Lambda)(\operatorname{resp} . \phi$ : $\operatorname{gr}_{G}(\Lambda) \rightarrow \bmod (\Lambda)$ ) is the forgetful functor and $X$ is an object in $\operatorname{gr}_{G *}(\Lambda)$ (resp. $\left.\operatorname{gr}_{G}(\Lambda)\right)$. By Theorems 3.4 and 3.2 there is a morphism $F:(\Gamma, \rho) \rightarrow\left(\Gamma_{0}, \rho_{0}\right)$ so that

$$
\begin{array}{ccc}
\operatorname{rep}(\Gamma, \rho) & \stackrel{E^{*}}{ } & \operatorname{gr}_{G}(\Lambda) \\
F \downarrow & & \downarrow \phi \\
\operatorname{rep}\left(\Gamma_{0}, \rho_{0}\right) & \approx & \bmod (\Lambda)
\end{array}
$$

where $E^{*}$ is as in Theorem 3.2. By Theorem 2.6 and Lemma 2.5 there exists a morphism of graphs with relations $F^{\prime}:\left(\Gamma^{*}, \rho^{*}\right) \rightarrow(\Gamma, \rho)$ such that

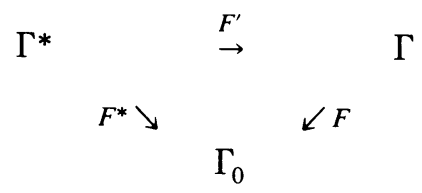


commutes. By Proposition 2.7 we get a commutative diagram:

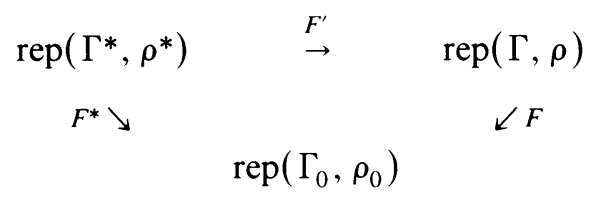

Apply Theorem 3.2 and the result follows.

The above result shows that the $G^{*}$-gradable $\Lambda$-modules are "universally" gradable modules (with respect to $\rho_{0}$ ). This concept is useful in the classification of Artin $k$-algebras of finite representation type (see [7]). The following example shows that different choices of $\rho_{0}$ yield different " universal" coverings.

EXAMPLE 4.2. Let $k$ be an algebraically closed field. Let $\Gamma_{0}$ be the finite directed graph

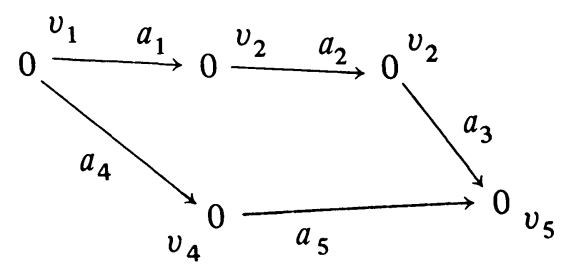

where $V\left(\Gamma_{0}\right)=\left\{v_{1}, \ldots, v_{5}\right\}$ and $A\left(\Gamma_{0}\right)=\left\{a_{1}, \ldots, a_{5}\right\}$. Let $T$ be the free path $k$-algebra associated to $\Gamma_{0}$. Let $\rho_{0}=\left\{a_{2} a_{1}, a_{5} a_{4}\right\}$ and $\rho_{0}^{\prime}=\left\{a_{2} a_{1}, a_{3} a_{2} a_{1}+a_{5} a_{4}\right\}$. Then $\rho_{0}$ and $\rho_{0}^{\prime}$ both generate the same ideal $I$ in $T$. Let $\Lambda=T / I$. Let $F_{1}:\left(\Gamma_{1}, \rho_{1}\right) \rightarrow\left(\Gamma_{0}, \rho_{0}\right)$ be the universal covering in $\operatorname{Cov}\left(\Gamma_{0}, \rho_{0}\right)$ and let $F_{2}:\left(\Gamma_{2}, \rho_{2}\right) \rightarrow\left(\Gamma_{0}, \rho_{0}^{\prime}\right)$ be the universal covering in $\operatorname{Cov}\left(\Gamma_{0}, \rho_{0}^{\prime}\right)$. Then, since $N\left(\Gamma_{0}, \rho_{0}, v_{1}\right)=\{\mathrm{id}\}, \Gamma_{1}$ is the simply connected universal covering $\Gamma_{0}$. Since $\pi_{1}\left(\Gamma_{0}, \rho_{0}, v_{1}\right)=\mathbf{Z}$, we conclude that there is a weight function $W: A\left(\Gamma_{0}\right) \rightarrow \mathbf{Z}$ such that the $\mathbf{Z}$-grading induced on $\Lambda$ is full and connected. For example,

$$
W\left(a_{i}\right)= \begin{cases}1, & i=1, \\ 0, & i \neq 0,\end{cases}
$$

works. In this $\mathbf{Z}$-grading on $T$, we see that $\rho_{0}$ is composed of homogeneous elements. On the other hand, since $a_{3} a_{2} a_{1}+a_{5} a_{4} \in \rho_{0}^{\prime}$, it follows that $N\left(\Gamma_{0}, \rho_{0}^{\prime}, v_{1}\right)=$ $\pi_{1}\left(\Gamma_{0}, v_{1}\right)$ and $\rho_{0}^{\prime}$ is not composed of homogeneous elements. Thus the identity map $\left(\Gamma_{0}, \rho_{0}^{\prime}\right) \rightarrow\left(\Gamma_{0}, \rho_{0}^{\prime}\right)$ is the universal covering in $\operatorname{Cov}\left(\Gamma_{0}, \rho_{0}^{\prime}\right)$. We conclude that for all groups $G$ there is no weight function $A\left(\Gamma_{0}\right) \rightarrow G$ such that the induced $G$-grading on $T$ is full and connected and the elements of $\rho_{0}^{\prime}$ are homogeneous in this grading.

We continue this example a bit further. Let $\Gamma_{1}$ be the graph with vertex set $\left\{\boldsymbol{v}_{i j}\right\}_{i=1, \ldots, 5 ; j \in \mathbf{Z}}$ and arrow set $\left\{a_{i j}\right\}_{i=1, \ldots, 5 ; j \in \mathbf{Z}}$ where $a_{i j}$ has origin

$$
\left\{\begin{array} { l l } 
{ v _ { i j } , } & { i = 2 , 3 , } \\
{ v _ { 1 , j } , } & { i = 1 , } \\
{ v _ { 1 , j } , } & { i = 4 , } \\
{ v _ { 4 , j } , } & { i = 5 , }
\end{array} \quad \text { and terminus } \quad \left\{\begin{array}{l}
v_{2, j+1,} i=1, \\
v_{3, j}, \quad i=2, \\
v_{5, j}, \quad i=3, \\
v_{4, j}, \quad i=4, \\
v_{5, j}, \quad i=5 .
\end{array}\right.\right.
$$


Let $\rho_{1}=\left\{a_{2 j+1} a_{1 j}, a_{5 j} a_{4 j}\right\}_{j \in \mathbf{Z}}$. Let $F: \Gamma_{1} \rightarrow \Gamma_{0}$ by $F\left(a_{i j}\right)=a_{i}$. Then the reader may verify that $F:\left(\Gamma_{1}, \rho_{1}\right) \rightarrow\left(\Gamma_{0}, \rho_{0}\right)$ is the universal cover in $\operatorname{Cov}\left(\Gamma_{0}, \rho_{0}\right)$. Let $L_{1}$ : $V\left(\Gamma_{0}\right) \rightarrow V\left(\Gamma_{1}\right)$ by $L_{1}\left(v_{i}\right)=v_{i 0}$. This lifting yields the weight function $W: A\left(\Gamma_{0}\right) \rightarrow \mathbf{Z}$ described earlier in this example. Now let $T=\amalg_{n \in \mathbf{Z}} T_{n}$ be the $\mathbf{Z}$-grading induced by $W$. Then $T_{0}$ is generated by the directed paths $v_{1}, \ldots, v_{5}$ (of length 0 ), $a_{2}, a_{3}, \ldots, a_{5}$, $a_{3} a_{2}, a_{5} a_{4} . T_{1}$ is generated by directed paths $a_{1}, a_{2} a_{1}, a_{3} a_{2} a_{1}$. Thus $T_{n}=0$ for $n \neq 0,1$. Thus the $\mathbf{Z}$-grading induced by $W$ on $\Lambda=\amalg_{n \in \mathbf{Z}} \Lambda_{n}$ has the property that $\Lambda_{0}, \Lambda_{1} \neq 0$, but $\Lambda_{n}=0, n \neq 0,1$. Now consider the lifting $L_{2}: V\left(\Gamma_{0}\right) \rightarrow V\left(\Gamma_{1}\right)$ by $L_{2}\left(v_{i}\right)=v_{i i}$. The weight function $W_{2}: A\left(\Gamma_{0}\right) \rightarrow \mathbf{Z}$ constructed in Theorem 3.2 for $L_{2}$ is given by

$$
W\left(a_{i}\right)= \begin{cases}1, & i=1,2,3,5, \\ 3, & i=4 .\end{cases}
$$

In this case the $\mathbf{Z}$-grading induced by $W_{2}$ on $T$ has $T_{0}$ generated by the paths $v_{1}, \ldots, v_{5}, T_{1}$ generated by $a_{1}, a_{2}, a_{3}, a_{5}, T_{2}$ generated by $a_{2} a_{1}, a_{3} a_{2}, T_{3}$ generated by $a_{4}, a_{3} a_{2} a_{1}, T_{4}$ generated by $a_{5} a_{4}$ and $T_{n}=0, n \neq 0,1,2,3,4$. Thus the Z-grading on $\Lambda=\amalg_{n \in \mathbf{Z}} \Lambda_{n}$ induced by $W_{2}$ has the property that $\Lambda_{0}, \Lambda_{1}, \Lambda_{2}, \Lambda_{3}$ are all $\neq 0$ and $\Lambda_{n}=0, n \neq 0,1,2,3$. Thus the $\mathbf{Z}$-gradings on $\Lambda$ constructed from the liftings $L_{1}$ and $L_{2}$ are different. As remarked earlier though, one may define a "graded equivalence" on $\Lambda$ in this case, a concept we will study elsewhere.

\section{REFERENCES}

1. Peter Gabriel, Auslander-Reiten sequences and representation-finite algebras, Representation Theory 1 (Dlab and Gabriel, Editors), Lecture Notes in Math., vol. 831, Springer-Verlag, Berlin and New York. 1980 .

2. Coverings in representation theory, Talk at ICRA III, Pucbla, Mexico, 1980.

3. R. Gordon and E. L. Green, Graded Artin algebras, J. Algebra 76 (1982), 111-137.

4. __ Representation theory of graded Artin algehras, J. Algebra 76 (1982), 138-152.

5. E. G. Green, Representation theory of tensor algebras, Thesis, Brandeis University, 1973.

6. ___ Representation theory of tensor algebras, J. Algebra 34 (1975), 136-171.

7. Group-graded algebras and the zero relation problem, Proc. ICRA III, Pucbla, Mexico, 1980 (to appear).

8. William S. Massey, Algebraic topologv: An introduction, Harcourt, Brace \& World, New York, 1967.

9. Christine Riedtmann, Algebren, Darstellungsköcher, Uberlagerungen and Zurück, Comment. Math Helv. 55 (1980), 199-224.

Department of Mathematics, Virginia Polytechnic Institute and State University, BlacksBURG, VIRGINIA 24061 